\title{
Design of a cost-effective customized Electronic Health Record system tohandle patient management during Covid-19 pandemic
}

\author{
Tasnim Ahmed, Saif Bin Sharif, Tahleel Abbas Joy, Moaz Hossain Chowdhury \\ and Mohammad Hasan Imam
}

\begin{abstract}
Healthcare is one of the basic needs for human beings. Unfortunately, a densely populated country like Bangladesh has always struggled to provide adequate healthcare to all of its people, especially to the rural population. To handle with huge amount of data and coordination between the doctors and patients, electronic health record system (EHR) has become a necessity. During this Covid-19 pandemic, face to face consultation between frontline workers like doctors, and nurse and patients has become difficult. As a result, many patients are having difficulty in getting treatment from doctors. In Bangladesh's perspective at this moment, a cost effective, energy efficient and portable device system is needed to build up a customized Electronic health record system. The proposed system can reduce medical errors about patient identification and treatment, increase effectiveness and timeliness of doctors and overall improve the health care of people of the rural area Therefore, an easy to use, affordable, user-friendly and customized EHR system design is proposed in this paper by utilizing recent wireless communication techniques that can give the rural people better health service during lockdown / isolated condition as imposed due to pandemic scenario.
\end{abstract}

Tasnim Ahmed was a student of Bachelor of Science in Computer Engineering, American International University- Bangladesh, Dhaka, Bangladesh.

Email: tasnim@ieee.org

Saif Bin Sharif was a student of Bachelor of Science in Computer Engineering, American International University- Bangladesh, Dhaka, Bangladesh.

Email: saififty007@gmail.com

Tahleel Abbas Joy was a student of Bachelor of Science in Computer Engineering, American International University- Bangladesh, Dhaka, Bangladesh.

Email: tahleeljoy@gmail.com

Moaz Hossain Chowdhury was a student of Bachelor of Science in Computer Engineering, American International University- Bangladesh, Dhaka, Bangladesh.

Email: moazchowdhury1234@gmail.com

Mohammad Hasan Imam is a Senior Assistant Professor of Department of Electrical and Electronic Engineering, American International UniversityBangladesh, 408/1, Kuratoli, Khilkhet, Dhaka 1229, Bangladesh

Email: hasan.imam@aiub.edu
Index Terms- Electronic Health Record (EHR), SQL, Raspberry pi-based computer, WebMD symptom checker

\section{INTRODUCTION}

According to International Organization of Standardization (ISO) in draft technical report, (ISO 2003), Electronic Health Record system (EHR) is the repository of information regarding the health of a subject of care (patient), in computer process able form, stored and transmitted securely, and accessible by multiple authorized users [1]. This information usually can be in the form of patient demographics, medical history, laboratory report, billing information and so on. EHR can be accessed by both the Doctors and the patients which provide various benefits like correct identification of patients, digital prescription which removes the readability problem of handwritten prescriptions, correct treatment plan determination by examining the previous medical history form a single file of the patient, artificial intelligence based Medical help for the patients which can prevent unnecessary visits to doctors where medical facility is very much available like many rural areas in under-developed and developing countries or in lockdown situations like Covid-19 pandemic scenarios.

Bangladesh badly needs Electrical Health Record system to cope up with this rapidly developing world and to build our digital Bangladesh. Our population is increasing day by day along with new problem and patients. To provide proper medical service to all the people with old days analog system

is quite impossible. EHR provides many advantages over traditional methods. In traditional method the record may get lost or damaged. Storing data can take up space for hospital and also searching, updating, inserting and deleting data is time consuming in traditional filing method. EHR can fasten up these processes by keeping data in digital format in data storage devices or in cloud data services. This will save labor and time and thus reducing the cost of healthcare. Electronic Health systems are designed to store data accurately and to capture the state of a patient across time. It eliminates the need to track down a patient's previous paper medical records and assists in ensuring data is accurate and legible [3, 5-12]. Providing proper health care to the rural area people always been a challenging job for our government. During the Covid19 pandemic it became one of the most important 
job for the authority. During the pandemic doctors are playing the most important role. But face to face interaction with patients put the doctors at the risk of getting infected. Face to face interaction always been playing an important role in medical sector and also patient prefer to meet the doctor or physician in person [2,3,]. But in this situation like covid-19 pandemic, face to face interaction should be the second, third or the last option. Nowadays many physician or doctors use the non-visit or virtual visit in some extent. To face the pandemic situation an improvised approach of Electronic Health Record system can make a huge difference. In Bangladesh's perspective, to implement electronic health record system using desktop computer or laptop would be a costly solution, whereas the country is already spending a huge amount of money on medical sector to survive the pandemic. In this case we can use single board computer like

- Raspberry pi, ASUS tinker board, NVIDIA Jetson nano developer kit (Specially designed for AI projects), Clockwork pi etc. Small computers are cost effective, has low energy consumption, portable and have enough power to run basic operations needed to maintain electronic medical data management operations. These small computers have enough processing speed to operate any electronic heath record system. By using these small computers energy consumptionby the processor can be reduced by 20 times [4]. Also, it can run longer than any laptop without electricity using the same battery backup which would be very helpful for the rural area. Therefore, Electronic health record system designed by using these small computers can improve the health care service for the rural area people as well as reduce the risk of getting affected by covid-19 virus. This paper proposes the design of a customized EHR in small scale for rural population which can be implemented in Bangladesh using the existing mobile network.

\section{METHODOLOGY OF THE PROPOSED SYSTEM}

In this pandemic Bangladeshi doctors are getting affected from covid-19 rate at a high rate. Doctors are the front liner who fight against the pandemic at the risk of their life. In recent times doctors are affected by the covid-19 due the patient's ignorance. In some cases, some hospitals are becoming partially or fully unserviceable for the same reason. To solve the problem health care system can be designed as such way that in-person meeting comes as second, third or the last option for the routine patient where the doctor can initially verify the patient's condition at that time. For this, a dedicated tent, separate office space can be used. In the other hand, in every Union Council there is a Union Health Services Council which is less crowded. The Union Health Services Council can have few telehealth devices to communicate with Sub District hospital or the district hospital. If a patient's symptoms lead to covid-19 positive, then healthcare worker can communicate with the doctor through the Telehealth device and talk to the doctor about the symptoms. Also, the Union Health Services Council can use dedicated space to verify the initial condition of the patients as discussed earlier.
At the time of emergency, Sub district or district hospitals can be informed through the device or Patient can use mobile application to contact the hospital. Also, in-ambulance testing system can be embedded with the tele-health system [8]. So that, the risk of getting affected will decrease and the hospital will get the time to take necessary steps to help the patient. In all these procedures in-person interaction with the doctor is always the last option.

To achieve that, a customized HER model is developed in this project which have two parts: one for the patient using a mobile application and the other for the doctors using Rasberry Pi based computing system and they are connected through wireless network for data storage and analysis.

The mobile application is built for the patients by which they can initially detect the symptoms of covid-19 using AI, where decision tree algorithm can be used [9]. In this project, we have embedded WebMD symptom checker to analyze patient disease symptoms which is integrated in the mobile app developed in Android platform and form that decision it can guide the patients next steps. WebMD symptom checker is free to use and effective [11]. The mobile application is created in android studio using java language. To develop the doctor's device interface .NET framework has been used. Moreover, using this app, patient can get emergency services like - Calling Ambulance, Emergency video/audio calling though app, inform the nearest hospital about the emergency and enquire about seat availability. Doctors are able to store the patient's data into the centralized hospital server after every consolation with the patients and the patients are able to see those data afterwards through the mobile application. The doctor's device and mobile application access a common database created using MySQL which contains the data stored by doctors. The database also contains the hospital seat availability and doctor's information. Figure 1and 2 show the overall block diagram and activity diagram system of the proposed system.

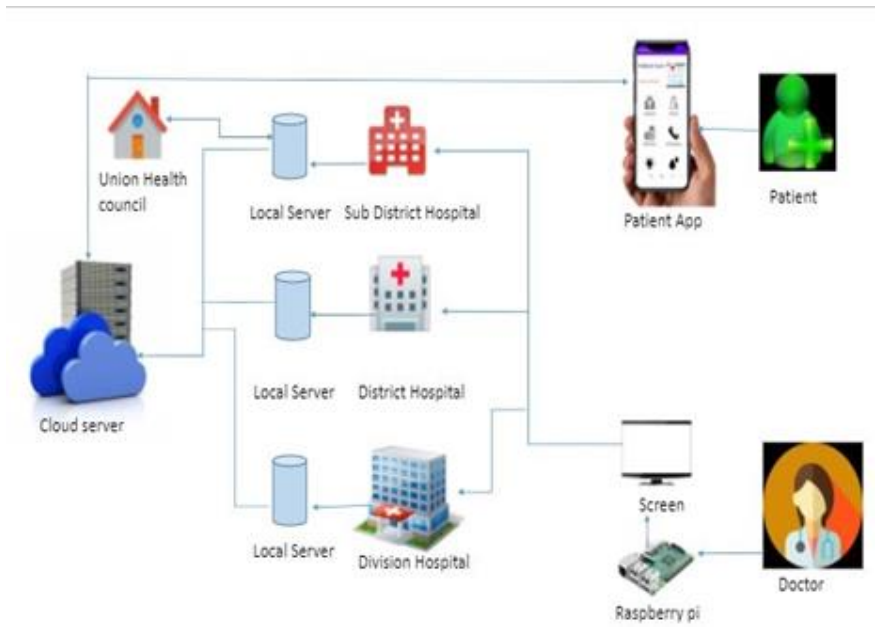

Fig. 1. Block diagram of the proposed EHR System 


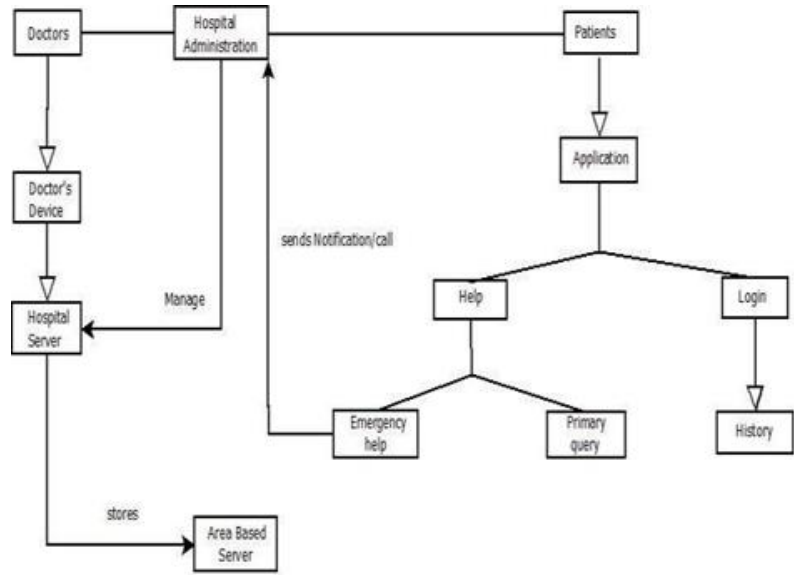

Fig. 2. Activity diagram of the proposed system

\section{A. Hardware Design}

To operate the whole system an operating device is designed for the doctors for data input and analysis. To build the device, Single Board Computer with attached display was used in this project. This single board computer's portability creates opportunities to use in different ways in different places according to our need and it's installation process is quite easy that, anyone with basic knowledge can install this small board computers.

For the single board computers Raspberry pi 4B model with 4GB variant was used. For the end user, Raspberry Pi 4 Model B provides desktop performance comparable to entry- level $\mathrm{x} 86$ PC systems [4]. This device's performance is comparable to entry level x86 PC system, which is enough Performance for run an electric health record system. Raspberry pi can be attached with any display through HDMI cable which also supports dual display. Therefore, this device easily can be used as a tablet or desktop computer, which will be much cheaper than any entry level laptop. The specification is given below with figure 3 .

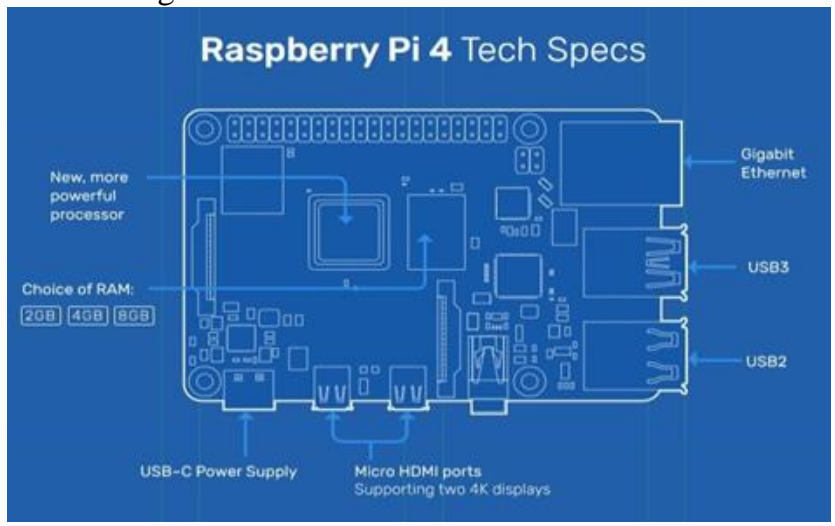

Fig. 3. Raspberry Pi 4 specifications [4]

Processor: Broadcom BCM2711, quad-core Cortex-A72 (ARM v8) 64-bit SoC @ 1.5GHz

Memory: 2GB, 4GB or 8GB LPDDR4 (depending on model)

Connectivity: $2.4 \mathrm{GHz}$ and $5.0 \mathrm{GHz}$ IEEE $802.11 \mathrm{~b} / \mathrm{g} / \mathrm{n} / \mathrm{ac}$ wireless LAN, Bluetooth 5.0, BLE Gigabit Ethernet 2 USB
3.0 ports 2 USB 2.0 ports.

GPIO: Standard 40-pin GPIO header (fully backwards compatible with previous boards)

Video sound: 2 micro HDMI ports (up to 4Kp60 supported) 2-lane MIPI DSI display port 2-lane MIPI CSI camera port 4pole stereo audio and composite video port - Multimedia: H.265 (4Kp60 decode); H.264 (1080p60 decode, 1080p30 encode); OpenGL ES, 3.0 graphics

SD card support: Micro SD card slot for loading operating system and data storage

Input power: 5V DC via USB-C connector (minimum 3A1), 5V DC via GPIO header (minimum 3A1) Power over, Ethernet (PoE)-enabled (requires separate PoE HAT)

\section{B. Software Design}

The designed software mainly acts as the heart of the electronic health record system. There are two parts of this software- 1. Doctor-end 2. Admin-end from the hospital or any other authorized user may be the local government health office. The admins are on the control of this system. They can add new doctor in the system, edit patient data, analyze data, response to the emergency need of the patients etc. as shown in figure 4. In the doctor's end, they can add new patients after logging in using their credentials like Name/ID and mandatory Password whose interface is shown in figure 5. Also, after the patient consultation, doctors add the data to the server and the patient get a digital copy of the prescription, and in future the patient data can be retrieved from the server. When a patient enters to the doctor's room, the doctor first tries to identify the patient by using the name or phone number or the NID/Birth Certificate number is shown in figure 6. If he/she is a new patient the doctor clicks on "New Patient" and add the identifying data in the server and proceeds further to the diagnosis/consultation. If he/she is an old patient, then the doctor clicks on "Old Patient" and login to the patient's account using the identifying number and proceeds to diagnosis. Also, doctor can see the previous history of a patient when doctor logs into the patient's account. [6-10]

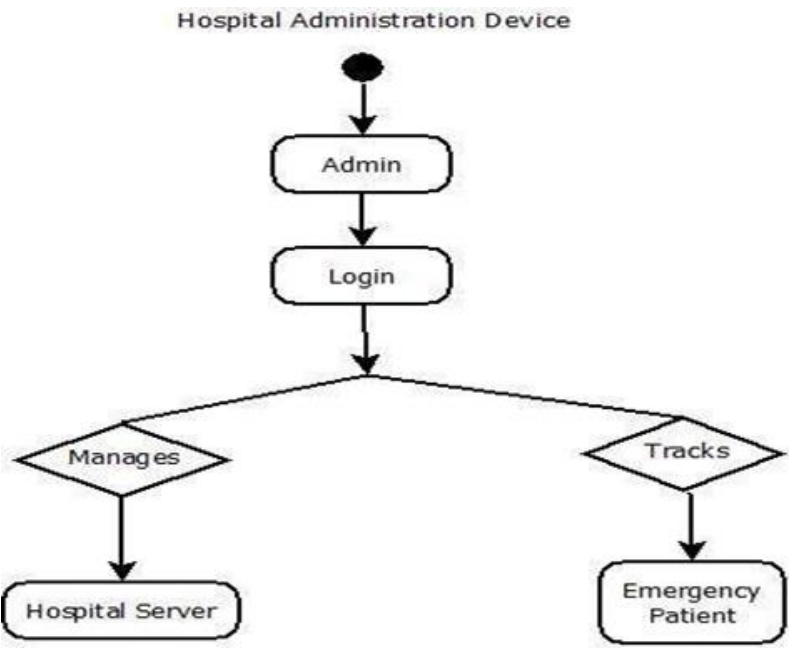

Fig. 4. Activity diagram Administration's device

.NET framework has been used to create the software and to 
create the pdf, iTextSharp has been used. To store the data Hosting database has been used. MySQL has been used to create the database.

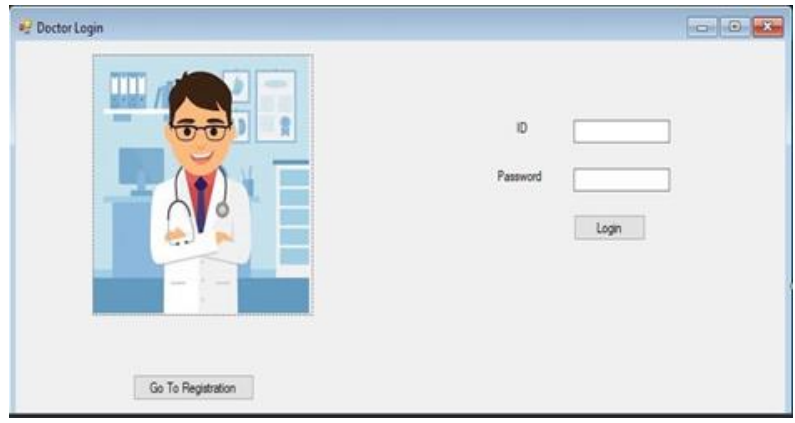

Fig. 5. Doctor Login interface

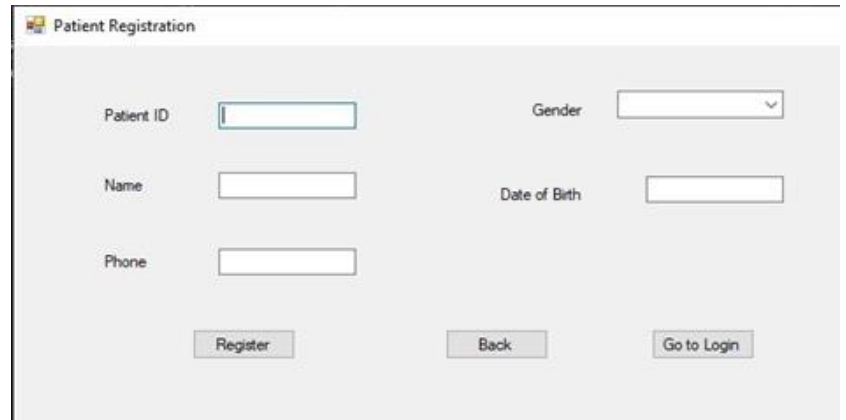

Fig. 6. Patient Registration interface

\section{PATENT MOBILE APPLICATION}

patient-end mobile application was created using android studio. Through the mobile application patient can see their previous diagnosis with a valid account by accessing the same database used to store data from doctor device. Also, patients can some other basic services through the application like Emergency contact with hospital, get hospital list, doctor list, audio calling service with the doctor etc. as shown in figure 7 .

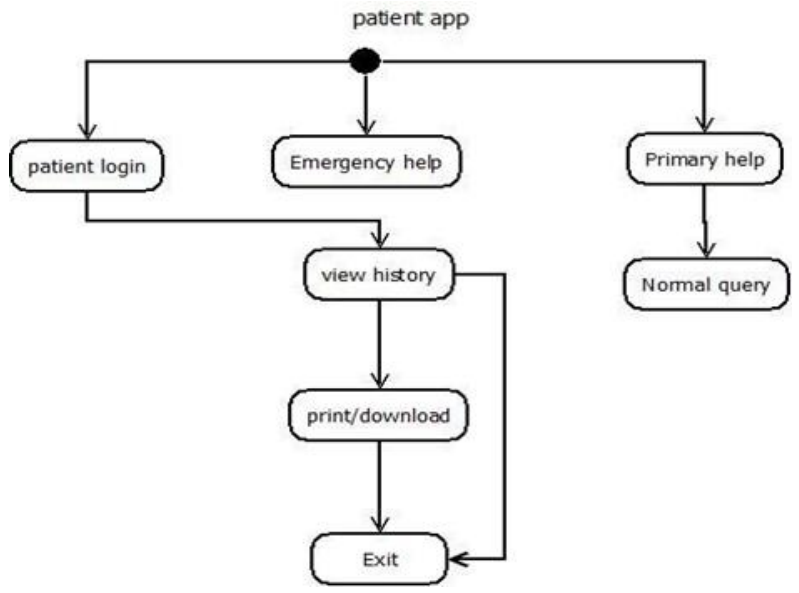

Fig. 7. Patient app activity diagram

To access to the patient's medical history through the mobile app, patient need to login to the system using username or phone number and password. From this mobile application people can access from everywhere around the world using the mobile network. A patient can get help anytime from this application. Patient can get available doctor's information, previous prescriptions/ medications prescribed by doctors, hospitals seat availability and nearest doctor's chamber details as shown in figure 8 . Our most important and helpful feature is emergency button. For any kind of emergency situation patient can directly call or send notifications to nearest hospital administration device and also call for ambulance or call police. Another feature is able to see hospital details. By clicking on hospital name patient can see hospitals details. Another most important feature is to get doctor information. By clicking on doctor's name people can see the doctor's details. A patient can consult with any doctor by calling them and can send images about personal condition or any scanned medical reports. Therefore, the doctor can see his reports and give him medical advice The Most interesting feature added is the symptom checker which can guide the patient about his health condition and his/her necessary action plan using artificial intelligence. Blood bank is another feature. In this feature we are adding blood donor list with contact information so that patient can easily find the donor details and call him/her in Emergency moment.

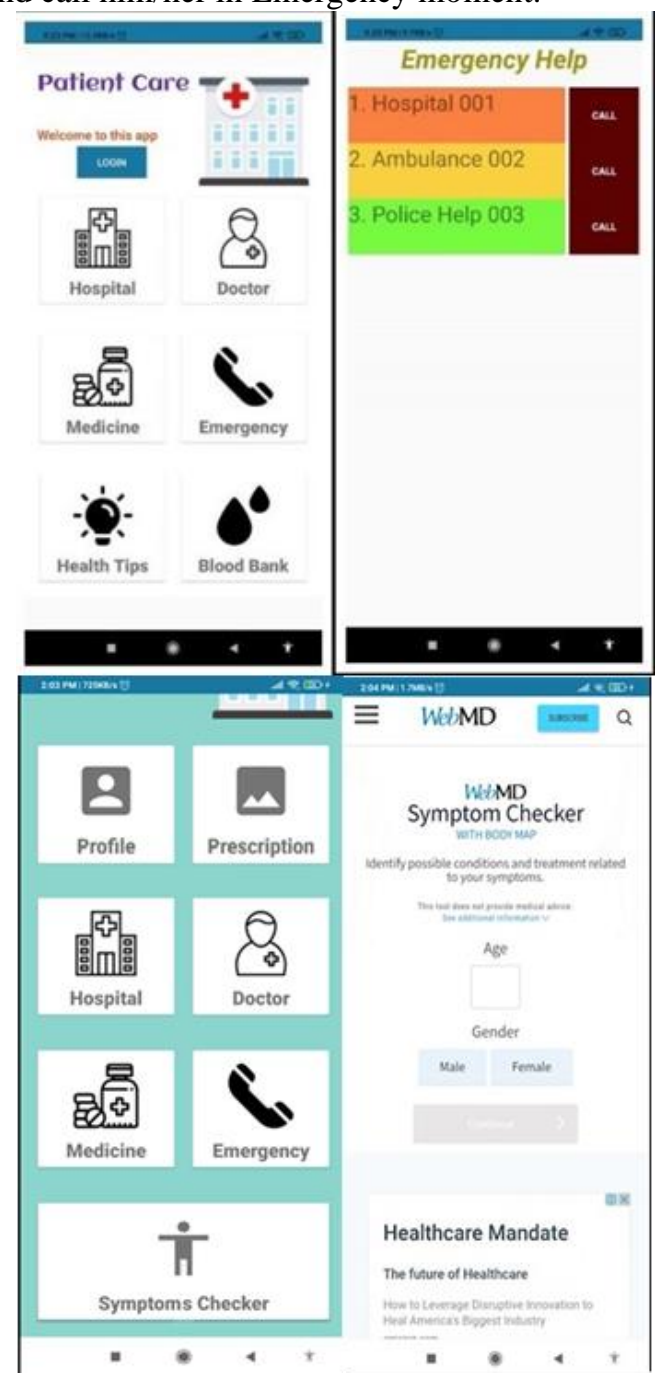

Fig.8. Patient app Features found after proper Login 
To make this app more user friendly we have added Medicine feature. In this feature we are adding medicine List with name, price and category. Patient can search medicine by giving name in search box and get details about medicine. Daily heath tips can also be found from the app form some selected newspaper link which is continuously updated as shown in figure 9.

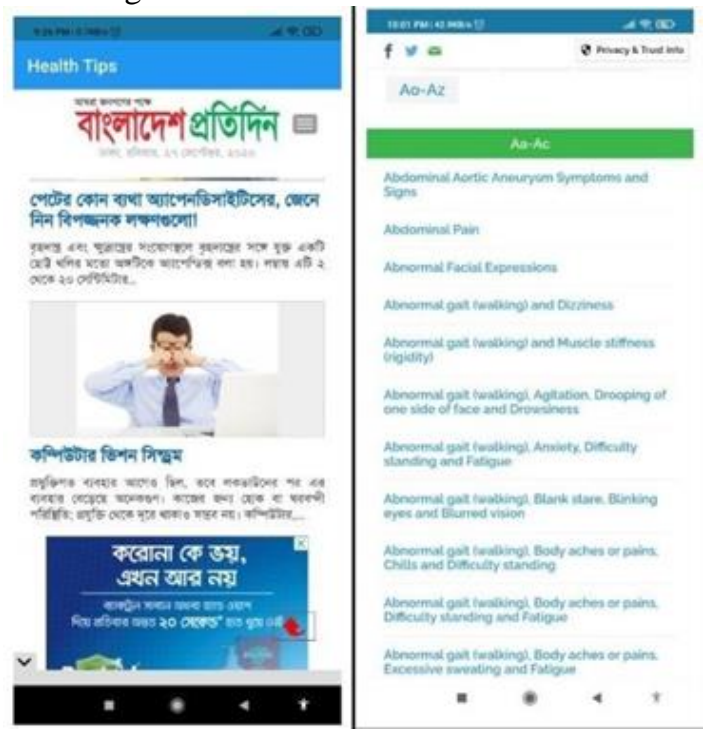

Fig.9. Health Tips Features added in the Patient app.

\section{DATABASE MANAGEMENT}

In this project, we have created 4 tables according to the ER diagram, where all the patient data are contained in one table as shown in figures 10 and 11. In the patient data table, doctor id and the hospital id use as foreign key to create the connection between hospital data table and doctor data table. There are 2 data tables for the hospital management which are patient data table, doctor data table. All the emergency contact and other service data which are provided by the admin are stored in the admin data table. In the database the union heath center would connect with sub-district hospital. The other hospitals in the sub-district area would connect with the subdistrict hospital database as well. In this process a central database has created.

\begin{tabular}{|c|c|c|c|c|c|}
\hline & d_id & password & d_name & gender & phone \\
\hline 1 & 1234 & ert 70 & Dr.Mahbub & Male & 01536105190 \\
\hline 2 & 3030 & lock 45 & Dr.Brishty & Female & 0186667727 \\
\hline 3 & 3450 & supes & Dr.nazrul islam & Male & 0178090111 \\
\hline 4 & 4321 & lock 21 & Dr. Triptyn & Female & 01742227621 \\
\hline \multicolumn{6}{|l|}{ - } \\
\hline & P_id & P_name & dob & gender & phone \\
\hline \multirow[t]{4}{*}{ • } & 10101 & Abdulah Mamun & 09/05/1990 & Male & 01966677727 \\
\hline & 20202 & Omar Fank & 13/12/1991 & Male & 01670238910 \\
\hline & 606 & Moaz & $09 / 12 / 2000$ & Male & 01536105190 \\
\hline & 909 & Mahmud & 1999 & Male & 01536105190 \\
\hline • & & & & & \\
\hline
\end{tabular}

Fig.12. Doctor and Patient data table example

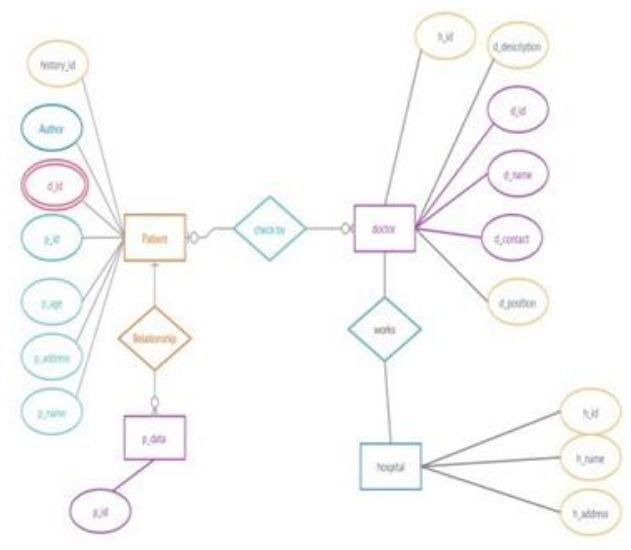

Fig. 11. ER diagram of the database

\section{DISCUSSION}

The project has been proposed for Bangladesh's perspective keeping in mind about a low cost, energy efficient and portable data handling device instead of traditional Desktop or Laptop system. We have tried to improve the Healthcare service through Digitalization and technology adoption. Doctors would interact with the patient using a Tablet computer. This would help the doctors to store the patient data permanently. A mobile application has been built for the patient so that they can easily fulfill their healthcare needs. Our prototype database is so simple, but to apply it in medical sector a lot off works needs to do. In our prototype we proposed an idea to overcome this pandemic in a smart way. Though our data are not categorized, we are able to build a

basic electronic record system for this pandemic situation. Now the world has many advanced technologies and also inventing new ones every day. These technologies should be embedded with the existing system to improve the healthcare system. To adopt with the pandemic situation fast, we need a system that can easily embedded. Also, there are few drawbacks. Still a lot of people are lacking knowledge about technology and introducing new technology in medical sector will a tough job for the government. Rural healthcare has always been a neglected field. One always has to work with limited budget and resources whenever working in a rural project. To improve the rural healthcare, more research and technological innovation is needed. Our project is a step towards that. It is important to show the scope of technological implementation in rural healthcare. If technological implementation shows improvement in rural healthcare, authorities will get inspired to allocate more resources in this field. We hope to attract more researchers to this field through our project. Rapid innovation can cut down the cost dramatically. This type of project will also improve the accessibility and affordability thus improving the participation. 


\section{REFERENCES}

[1] "ISO/TR 20514:2005", ISO, August 2020.

[2] Judd E. Hollander, M.D. and Brendan G. Carr, M.D. "Virtually Perfect? Telemedicine for Covid-19". New England Journal of Medicine, August 2020, pp.1679-1681

[3] Fletcher H. Kaiser's Tyson "Health care's future isn't in a hospital" Tennessean.com, April 21, 2017

[4] Akshay, S., Vishnukumar, B., Mohan, V. and S. Anand, M.,. "Energy and Performance Analysis of Raspberry Pi with Modern Computing Devices", International Journal of Engineering Technology, 2018 ,pp.777.

[5] A. Kumar, H. K. Jamnadas, A. B. M. S. Ali and J. Nahar, "Electronic Health Record: A Cost Effective Model for Developing Economies," 2018 5th Asia-Pacific World Congress on Computer Science and Engineering, 2018, pp. 214-220.

[6] Bhavnani, Sanjeev P, Narula, Jagat, Sengupta, Partho P. (7 May 2016)."Mobile technology and the digitization of healthcare" EuropeanHeart Journal, 7 May 2016 , pp.1428-38.

[7] Keyani, A. Mumtaz, H. Mushtaq and A. Hussain, "Affordable and accessible Tele-healthcare to rural areas of Pakistan through web and mobile based technologies" 2009 6th International Symposium on High Capacity Optical Networks and Enabling Technologies (HONET), Alexandria, 2009, pp. 110-114.

[8] Khan, Rida \& Zardari, Asad \& Bhatti, Zeeshan,"Artificial Intelligence based Smart Doctor using Decision Tree Algorithm", ITB Journal of Information and Communication Technology, 2017, pp. 1-5.

[9] M. Ginsburg, "Pediatric Electronic Health Record Interface Design: The PedOne System", 2007 40th Annual Hawaii International Conference on System Sciences, 2007.

[10] M. Sakib, M. Jaladeen and M. Razi, "Electronic Health Record System (EHR) Adoption: From Bangaladesh Medical Students' Perspective", 2015

[11] WebMD, "Symptom Checker from WebMD. Check Your Medical Symptoms.,"WebMD, 2019. https://symptoms.webmd.com/.'

[12] M. Alyami and Y. Song, "Removing barriers in using personal health record systems", 2016 IEEE/ACIS 15th International Conference on Computer and Information Science (ICIS), 2016.

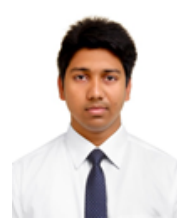

Tasnim Ahmed was admitted in Bachelor's in science degree in 2017 at American International University-Bangladesh and completed his B. Sc. Engg. in Computer Engineering in December 2020. Currently, he is working in an USA based home care service provider company. His research interest includes biomedical informatics and biomechanics.

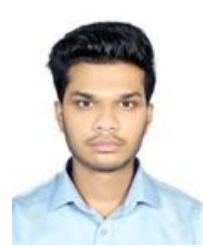

Saif Bin Sharif passed his SSC and HSC from Adamjee Cantonment Public School and College in 2014 and 2016 respectively. He finished his bachelor's degree in Computer Engineering (CoE) from American International University-Bangladesh (AIUB) in January 2021. His research interests includes Data Mining, Health Technology and Network security.

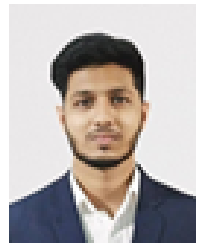

Tahleel Abbas Joy passed his SSC from Comilla Modern Hight School in 2014 and HSC from Cumilla Biggan College in 2016. He finished his bachelor's degree in Computer Engineering (CoE) from American International University-Bangladesh (AIUB) in October 2020. Now he is working with a American Homecare agency. His main research interests include different topics Like Machine Learning, Web Developing, Mobile Application Making etc.

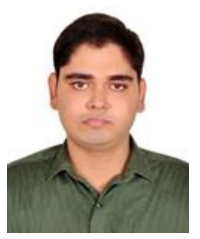

Moaz Hossain Chowdhury was born in Dhaka, Bangladesh. He has completed B.Sc Computer Engineering from American International University Bangladesh. His research interests include Machine Learning, Data Mining and Health Technology. He is currently working for a Business Enterprise.

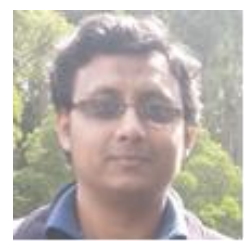

Mohammad Hasan Imam (MIEEE) Completed his B.sc Engg. from Bangladesh University of Engineering and Technology (BUET) in 2004 and Completed his $\mathrm{PhD}$ in 2015 from the University of Melbourne, Australia in the area of signal Processing especially Bio signal Processing and Modeling. He is currently working as Senior Assistant Professor in the department of Electrical and Electronic Engineering at the American International University-Bangladesh (AIUB). His main research interests include different topics in the area of bioengineering like Bioinformation processing, Model base system dynamics analysis, Machine learning in healthcare, Biomedical signal processing and Biomedical electronics. 